\title{
Caracterización de la Responsabilidad Profesional Medica, Dirección de Medicina Forense de
} Tegucigalpa.

Characterization of complaints of medical professional responsibility, issued by Dirección de Medicina Forense in Tegucigalpa.

Mayely Cedillo ${ }^{1 *}$, Gustavo Roque Pacheco ${ }^{2}$, Mireya Matamoros ${ }^{3}$

\author{
https://doi.org /10.5377/rcfh.v5i2.8862
}

${ }^{1}$ Posgrado Medicina Legal y Forense, Facultad de Ciencias Médicas, Universidad Nacional Autónoma de Honduras; ${ }^{2}$ Unidad de Mala Praxis, Dirección de Medicina Forense Tegucigalpa, ${ }^{3}$ Investigación y Docencia en Ciencias Forenses, Dirección de Medicina Forense Tegucigalpa y Hospital San Felipe.

*Correspondencia a Mayely Cedillo: mayelycedillo@gmail.com

\section{CITAR COMO}

Cedillo M, Pacheco GR, Matamoros M. Caracterización de la responsabilidad profesional médica en Medicina Forense de Tegucigalpa. Rev. cienc. forenses Honduras. 2019; 5(2):3-13.

\section{ASPECTOS ÉTICOS}

El presente estudio contó con el aval de las autoridades de la Dirección de Medicina Forense para la revisión de las denuncias, se guardó el anonimato de los denunciados y denunciantes.

Los autores declaran que no hay conflicto de interés en la publicación de este artículo.

RECIBIDO: Nov. 2018 ACEPTADO: Junio 2019

\section{RESUMEN:}

Justificación: Las fallas relacionadas a la actuación profesional, impactan negativamente a los pacientes e incluso pueden conducirlos a la muerte. Las denuncias constituyen un mecanismo por el que los pacientes o sus familiares expresan insatisfacción, y cuando se analizan, sistemáticamente, no solo desde la perspectiva legal, constituyen una oportunidad de mejora continua. Objetivo: Caracterizar las denuncias por responsabilidad profesional médica dictaminadas en la Dirección de Medicina Forense de Tegucigalpa. Metodología: Se realizó un estudio descriptivo de 89 denuncias por responsabilidad profesional médica, entre el 2010 y el 2015 dictaminadas en la Dirección de Medicina Forense de Tegucigalpa, siendo las variables de estudio: $\underline{\mathrm{De}}$ las víctimas: edad, sexo, escolaridad. De los denunciantes: edad, sexo, motivo de la denuncia, relación con la víctima, procedencia. Del denunciado: Edad, sexo, centro donde laboraba, tiempo de laborar y especialidad ejercida. Resultados: El $5,61 \%$ de las denuncias correspondieron a casos de responsabilidad profesional médica, $20 \%$ de las cuales fueron por desconocimiento inexcusable y $80 \%$ por omisión de cuidados. Ginecobstetricia fue la especialidad más denunciada con el $30 \%$. Los médicos más denunciados $(77,6 \%)$ trabajaban en una institución pública, y el conflicto en la relación médicopaciente fue la causa de denuncia más frecuente (40.4\%). Conclusión: Los médicos del sexo masculino, cercanos a los 50 años de edad y con más de 15 años de experiencia, que laboraban en centros públicos y que ejercían las especialidades de Ginecobstetricia, Ortopedia y Cirugía General, 
fueron los más denunciados; entre los motivos más frecuentes de denuncia se citaron los conflictos en la relación médico-paciente-familiar, la inconformidad en el diagnóstico y falta de ética profesional de otros profesionales con comentarios adversos hacia sus colegas o las instituciones.

\section{PALABRAS CLAVE}

Responsabilidad profesional, responsabilidad legal, negligencia, impericia.

\section{ABSTRACT}

Introduction: The failures related to the professional performance, have a negative impact on the patients and in some cases lead to their death. Complaints are a way for patients and their families to express dissatisfaction with the service received, as well as an opportunity for continuous improvement if they are analyzed, not only from a legal perspective. Objective: To characterize of complaints of medical professional responsibility issued by the Dirección de Medicina Forense in Tegucigalpa. Methodology: A retrospectivedescriptive study of 89 complaints for medical professional responsibility of live and dead subjects was conducted during the period 2010 to 2015 by the Dirección de Medicina Forense in Tegucigalpa, considering the factors: From the victims: Age, sex, education. From the complainant: Age, sex, reason for complaint, relation to the victim, place of origin. From the accused: Age, sex, workplace, employment history, medical specialty. Results: Only $5.61 \%$ of the complaints corresponded to cases of medical professional responsibility, $20 \%$ of those were due to inexcusable lack of knowledge and $80 \%$ due to medical care omission. Gynecoobstretics was the most reported specialty with $30 \%$. Of those accused, $77.6 \%$ worked in a Public institution, the conflict in the doctor-patient relationship was the most frequent cause of complaint (40.4\%). Conclusions: The characteristics of the allegations studied show that doctors who were close to 50 years old and had more than 15 years of experience, who worked in public centers received the most complaints, gyneco-obstretics, orthopedics and general surgery, were the specialties that received most of complaints; The most common complaints were related to the doctor-patient-family relationship, the disagreement with the diagnostic and the lack of professional ethic were adverse comments directed towards colleagues or the institutions.

\section{KEYWORDS}

Professional responsibility, legal responsibility, negligence, Unproficiency.

\section{INTRODUCCIÓN}

La responsabilidad profesional médica es la obligación que tiene toda persona que ejerce la medicina de responder ante la justicia por los daños ocasionados con motivo del ejercicio de su profesión ${ }^{1-3}$, la Organización Mundial de la Salud (OMS) estima que a escala mundial, cada año decenas de millones de pacientes sufren lesiones discapacitantes o mueren como consecuencia de prácticas médicas o atención inadecuada, sumado a los costos de atención y pago de indemnizaciones que conllevan 4,5 , por tanto su análisis es importante y constituye una herramienta de mejora continua si se estudian, no solo desde el ámbito legal punitivo, sino desde la perspectiva de mejora continua.

En nuestro país poco se conoce sobre la dinámica y características de los casos de responsabilidad profesional médica, por lo que, para conocer mejor este problema, se caracterizaron las denuncias dictaminadas en la Dirección de Medicina Forense de Tegucigalpa, durante el periodo 2010-2015.

En Honduras las denuncias por responsabilidad profesional son recibidas por las Fiscalías y trasladadas a la Dirección de Medicina Forense 
para su análisis pericial ${ }^{6}$, y al igual a lo que ocurre en otros países, no hay legislación especial que regule la responsabilidad profesional médica, por lo que los juicios suelen encasillarse dentro de las figuras de delito culposo, siendo este el resultado de imprudencia, impericia, negligencia o cuando es producto de la inobservancia de una ley, de un reglamento o de órdenes ${ }^{7}$.

En los casos de responsabilidad profesional medica no solo se asume responsabilidad penal, sino también responsabilidad civil, la cual implica la obligación jurídica de la reparación económica del daño causado por responsabilidad profesional médica. (Art. 2236 Código Civil de Honduras) ${ }^{8}$. El Colegio Médico de Honduras también establece sanciones para el médico en su Reglamento de Sanciones ${ }^{9}$.

Otro tipo distinto se encuentra en el ámbito contencioso- administrativo en el que no se pone en tela de juicio la actuación concreta del médico, sino de la administración sanitaria. Con frecuencia se trata de analizar si se han puesto al alcance del paciente los medios necesarios en función de las características del centro, este supuesto se trata de una cuestión meramente económica ${ }^{3}$.

\section{MÉTODOLOGÍA}

Se realizó un estudio retrospectivo-descriptivo de 89 denuncias por responsabilidad profesional médica, interpuestas ante la Fiscalía y remitidas a la Dirección de Medicina Forense de Tegucigalpa, para su análisis pericial, durante el periodo 2010 al 2015. Para fines de este estudio se estableció como denuncia, el expediente médico legal conformado por la denuncia, la trascripción literal del expediente clínico, los estudios complementarios y el dictamen pericial. Setenta y nueve (79) de las denuncias analizadas correspondieron a sujetos vivos y 10 a personas muertas. Se excluyeron del análisis, las que aún no habían sido dictaminadas, las correspondientes a responsabilidad profesional no médica, y aquellas cuyo proceso judicial continuaba abierto al momento del estudio.

\section{Procesamiento y análisis de datos:}

La información fue recolectada en un instrumento construido en Microsoft Excel $2016{ }^{\circledR}$ y codificada para mantener el anonimato. Las variables estudiadas fueron:

- Sociodemográficas del afectado y denunciante (Edad, sexo, ocupación, procedencia, escolaridad, motivo de denuncia, relación con el afectado).

- Sociodemográficas del denunciado (Edad, sexo, años de laborar, especialidad médica desempeñada).

- Tipos de denuncia: Con o sin responsabilidad profesional desde el punto de vista médico legal.

- $\quad$ Causa de responsabilidad: Se enumeraron las causas de responsabilidad profesional medica según el análisis pericial médico forense.

La información se analizó con el programa SPSS ${ }^{8}$.

\section{RESULTADOS}

El 5,61\% (5/89) de las denuncias correspondieron a casos de responsabilidad profesional médica, el $94.38 \%$ (84/89) se dictaminaron como casos sin responsabilidad profesional médica, desde el punto de vista médico legal. Las causas por las que se incurrió en responsabilidad profesional médica se detallan en el Cuadro 1.

Cuadro 1: Causas de responsabilidad profesional médica según dictamen médico legal

\begin{tabular}{|c|c|c|c|}
\hline Causas & & $\mathbf{N}^{\circ}$ & Porcentaje \\
\hline $\begin{array}{l}\text { Desconocimiento } \\
\text { justificado }\end{array}$ & no & 1 & 20 \\
\hline Omisión de cuidados & & 4 & 80 \\
\hline Total & & 5 & 100 \\
\hline
\end{tabular}

Fuente: Registros de la Dirección de Medicina Forense, Tegucigalpa 
Cuadro 2. Distribución de denuncias dictaminadas sin responsabilidad profesional medica

\begin{tabular}{lcc} 
Resultado & Frecuencia & Porcentaje \\
$\begin{array}{l}\text { Responsabilidad } \\
\text { estatal }\end{array}$ & 1 & 1,19 \\
$\begin{array}{l}\text { Denuncias sin } \\
\text { argumento }\end{array}$ & 34 & 40,47 \\
latrogenias & 49 & 58,33 \\
Total & $\mathbf{8 4}$ & 100 \\
\hline
\end{tabular}

Fuente: Registros de la Dirección de Medicina Forense,

Tegucigalpa.

Respecto a las denuncias que se dictaminaron sin responsabilidad profesional médica, su distribución se muestra en el Cuadro 2, entendiéndose como "denuncias sin argumento" aquellas que se motivaron en el límite del conocimiento del paciente y que después de su análisis no se encontró fundamento científico-disciplinar.

Se determinó que el $22,64 \%$ de las denuncias eran contra centros privados y el $76,34 \%$ contra centros públicos, siendo los centros públicos del sistema de Seguridad Social los más denunciados con el 31,5\%.

Las especialidades más denunciadas fueron Ginecobstetricia (30,0\%), Ortopedia (14,61\%), Cirugía General (13,48\%) y Pediatría (11,24\%). La distribución de denuncias por especialidad se muestra en el Cuadro 3.

El $59,6 \%$ de los denunciados son masculinos y $23,6 \%$ son femeninos, en el $16.8 \%$ de los casos no fue posible establecer el sexo.

En promedio los médicos más denunciados poseen una edad cercana a los 50 años y tienen más de 15 años de experiencia, como muestra el Cuadro 4.

Respecto a los afectados El 62.9 \% procedían del área urbana de Tegucigalpa, el 37,1\% del área rural. El 53,9\% de los denunciantes fueron los mismos afectados, en el $28,1 \%$ los padres y en el $12,4 \%$ sus
Cuadro 3. Distribución de denuncias por especialidad médica

\begin{tabular}{lcc}
\hline Especialidad & Frecuencias & Porcentaje \\
\hline Ginecobstetricía & 27 & 30.34 \\
Ortopedía & 13 & 14.61 \\
Cirugía general & 12 & 13.48 \\
Pediatría & 10 & 11.24 \\
Medicina general & 7 & 7.87 \\
Neurocirugía & 4 & 4.49 \\
Oftalmología & 3 & 3.37 \\
Radiología & 3 & 3.37 \\
Otras & 10 & 11.24 \\
Total & 89 & 100.00
\end{tabular}

Fuente: Registros de la Dirección de Medicina Forense, Tegucigalpa

parejas sentimentales.

Los motivos, según el denunciante, que originaron la denuncia se muestran en el Cuadro $\mathrm{N}^{\circ} \mathbf{5}$, siendo el más frecuente el conflicto en la relación médico paciente-familiar con el $40 \%$, la inconformidad con el diagnóstico $20,2 \%$ y la falta de ética profesional, cuando se realizan comentarios adversos sobre otro colega o contra la institución en el 12,4\%.

\section{DISCUSIÓN}

Los resultados indican que el 5,61\% (5/89) de las denuncias se dictaminaron con responsabilidad profesional médica; el $20 \% \quad(1 / 5)$ por desconocimiento no justificado, relacionada con la figura legal de impericia y el 80\% (4/5) por omisión de cuidados relacionada a la figura legal de negligencia médica.

Con respecto a la conducta omisiva (por ejemplo, casos de diagnóstico fallido), el dictamen pericial omisión y el evento (daño o lesión) configurable hipotéticamente, de que, si se hubiesen tomado 
medidas necesarias, el daño o lesión no habría tenido lugar, o habría tenido lugar sustancialmente más tarde, o con una intensidad menos perjudicial. Según el código penal hondureño, la impericia (falta de habilidad), consiste en una mala actitud en aquellas actividades que requieren un conocimiento técnico especial. Por ejemplo, cuando el médico no alcanza las habilidades mínimas y experiencia técnica en el uso de instrumentos, o en el diagnóstico erróneo de un caso "fácil"; entendiéndose como el procedimiento médico (quirúrgico ó no) rutinario, comúnmente realizado y directo, 7,10.

La negligencia consiste en la falta de atención e implica comportamiento pasivo que se refleja en la omisión de las precauciones necesarias. Por ejemplo, cuando el cirujano deja clips, instrumentos o esponjas de gasa en la herida quirúrgica, opera el miembro sano, no controla la fecha de vencimiento de los medicamentos o no realiza las investigaciones preliminares necesarias 7,10 .

Cuadro: $N^{\circ}$ 4: Edad y Experiencia Laboral de los denunciados.

\begin{tabular}{lcccccccc} 
& $\mathbf{n}$ & Min & Cuartil1 & Sd & Media & Mediana & Cuartil3 & Max \\
Edad del denunciado & 74 & 35 & 45 & 9.17 & 50.15 & 50 & 55.0 & 75 \\
Experiencia laboral & 74 & 2 & 10 & 8.24 & 16.38 & 15 & 21.5 & 35 \\
\hline
\end{tabular}

Fuente: Registros de la Dirección de Medicina Forense de Tegucigalpa

\section{Cuadro $\mathrm{N}^{\circ}$ 5: Motivos de las denuncias según el denunciante}

\begin{tabular}{lcc} 
Causas & Frecuencias & Porcentaje \\
\hline Conflicto en la relación médico-paciente-familiar & 36.0 & 40.4 \\
Demora en consulta o sala & 8.0 & 9.0 \\
Inconformidad en el diagnóstico & 18.0 & 20.2 \\
Falta de ética profesional & 11.0 & 12.4 \\
Falta de infraestructura, materiales y/o equipo del centro & 5.0 & 5.6 \\
asistencial & & \\
Motivación por indemnización económica & 11.0 & 12.4 \\
Total & 89 & $\mathbf{1 0 0 . 0}$
\end{tabular}

Fuente: Registros de la Dirección de Medicina Forense de Tegucigalpa 
En Estados Unidos en un estudio realizado en 1991 analizando 31,429 pacientes hospitalizados, se encontró una tasa ponderada de responsabilidad profesional de 1,53 por ciento; (IC 95\%, rango entre 0 a 3,2\%)11, si bien es cierto el número de denuncias va en aumento, no ha sido posible determinar la magnitud real del problema en virtud que muchas de las demandas se arreglan extrajudicialmente, además de los problemas de registro y análisis de la información, que constituye una limitante importante.

Entre las causas más comúnmente descritas por las que los médicos incurren en errores están: Los problemas de comunicación con los colegas, pacientes y familiares, deficiencias en educación, entrenamiento, y experiencia, la sustitución del método diagnóstico, por el uso de recursos tecnológicos ${ }^{11,12}$, el uso de exámenes diagnósticos obsoletos, retraso diagnóstico y médicos practicando fuera de su campo de experticia ${ }^{13}$, realizar la atención de manera automática, sin buscar la opinión de otros colegas, evaluación incompleta al ingreso, no obtener el consentimiento del paciente o de los familiares 14,15, exceso de trabajo, fatiga, estrés, mala actitud, delegación de responsabilidades a personal con competencias insuficientes, desmotivación, falta de actualización, exceso de confianza ${ }^{22,23}$. Un estudio realizado en Reino Unido en sentencias por fallas en la responsabilidad profesional identificó cuatro factores de riesgo a priori: (1) ser hombre, (2) haber obtenido su licencia médica primaria fuera del Reino Unido, (3) trabajar dentro de la práctica general y especialidades quirúrgicas, y (4) haber aprobado su licenciamiento medico hace más de 20 años, también señalaron otros factores como el aumento de la edad y la falta de orientación clínica ${ }^{18}$.

Institucionalmente se ha descrito la incapacidad para indicar estudios complementarios adecuados y actuar según los resultados de estos; políticas inadecuadas, falta de consistencia en los procedimientos institucionales, personal de apoyo escaso o inadecuado y supervisión deficiente; las fallas técnicas asociadas con el equipo médico, el que no se disponga o promueva la cultura de registro o informe de errores y eventos adversos 14,15 .

En nuestro estudio la información disponible no permitió identificar las causas que condujeron a incurrir en responsabilidad profesional.

En el $94,38 \%$ de las denuncias no se determinó responsabilidad profesional médica, encontrándose que el 1.19\% (1/84) correspondió a responsabilidad institucional (sistema de salud), lo que en España se ha descrito como "Pérdida de la oportunidad asistencial" ${ }^{19}$, el 40,47\% (34/84) no tenían argumento y 58,04\% (49/84) correspondieron a iatrogenias.

Según el diccionario de la Real Academia Española, "iatrogenia" es una alteración, de connotación negativa del estado del paciente, es decir, aquel daño que genera el médico, derivado del método diagnóstico, terapéutico, medicamentoso, o como el acto que desencadena una complicación, patología o muerte pero que no le es imputable judicialmente al médico ${ }^{20,21}$. Si bien es cierto la iatrogenia no conlleva imputabilidad penal del profesional, esta debe ser valorada desde el enfoque de la seguridad del paciente con el fin de evitarle daños. La iatrogenia debe reconocerse como un importante problema de salud pública, que implica en algunos casos daños irreversibles al paciente; en nuestro país la iatrogenia sigue siendo un tema aun sin explorar.

Respecto a los motivos de denuncia se encontró que el conflicto en la relación médico-pacientefamiliar, fue el motivo más importante, con el $40.4 \%$, lo que concuerda con lo reportado por otros 
autores, que indican que el factor fundamental por el que se denuncia tiene que ver con una mala comunicación médico-paciente, en cuanto a su diagnóstico, estudios, tratamiento, pronostico etc.; y la sensación del paciente que no ha sido adecuadamente informado ${ }^{18,22-25}$.

El 20.2\% denunció debido a la inconformidad en el diagnóstico, el $12.4 \%$ denunció debido a la influencia que los comentarios negativos de otros galenos, realizan contra sus colegas o contra la institución. La opinión de otro médico influye en el índice de demandas. Los pacientes informan sobre este tema: "Sí, otro doctor ya me dijo que eso estaba mal hecho, que era una barbaridad lo que me hicieron"23.

En un estudio realizado en la Universidad de Guadalajara en el año 2014; la mayor cantidad de quejas estaba relacionada con tres asuntos: una mala relación médico-paciente, una mala operación del sistema de salud y un mal diagnóstico ${ }^{25}$.

Nuestros resultados indican que los médicos que laboran en los centros públicos son los más denunciados (76.34\%) en contraste con lo descrito por Tamara y colaboradores (Bogotá 2012), que encontró que los prestadores de salud privados representaron el 51,99\% de los casos y los centros públicos el 43,28\%26, este predominio de centros públicos versus privados encontrado en nuestra caracterización podría explicarse en relación a aspectos institucionales como la organización y diseño de actividades, las condiciones de trabajo (sobrecarga laboral, la no sistematización de los procesos), la falta de supervisión, la escasa comunicación, el pobre equipamiento y mantenimiento del equipo y de las instalaciones, sumado a la escasa cultura de enfoque de "servicio y respeto al paciente" que suelen tener los servidores públicos del sistema de salud en Honduras.
Respecto a las especialidades que ejercen los médicos más denunciados son la ginecobstetricia con el $30 \%$, Ortopedia con el $14,61 \%$ y Cirugía General con el $13,48 \%$ coincidiendo con lo reportado, indicando que Ortopedia y Traumatología, Ginecología y Obstetricia fueron las especialidades con mayor número de reclamaciones ${ }^{10,27}$.

Asimismo, Castellanos, en el 2014 reporto que entre las 10 especialidades médicas más reclamadas en Europa estaban: Ginecología y Obstetricia (13.5\%), Cirugía Ortopédica (12:3/\%), Urgencias (9.7\%), siendo las causas más comunes de responsabilidad profesional la complicación postoperatoria; falla de diagnóstico; consentimiento inadecuado y cirugía en el sitio equivocado 28,29 .

Los médicos de mediana edad (50años), con 15 años de experiencia en promedio son los más denunciados, resultados similares reportaron estudios realizados en estados Unidos, Reino Unido y Colombia ${ }^{18,30,31}$. Estos resultados podrían explicarse por el exceso de confianza del profesional, y el desgaste laboral asociado a más de una década de servicio.

El $59,6 \%$ de los denunciados son masculinos y $23,6 \%$ son femeninos, en el $16.8 \%$ de los casos no fue posible establecer el sexo. Este hallazgo concuerda con lo reportado en otros estudios; en una revisión sistemática realizada en Reino Unido para examinar la relación entre el sexo y las demandas por responsabilidad profesional se encontró en 27/32 estudios que los médicos varones tenían más probabilidades de haber experimentado una denuncia por responsabilidad profesional. Los resultados mostraron que los médicos varones tenían casi dos veces y media más probabilidades de ser sujetos a una acción médico legal que las mujeres. Hubo un efecto general significativo del sexo en cada estrato, ya que los 
médicos varones tuvieron mayores probabilidades de denuncia por responsabilidad profesional en América del Norte: (OR, 2.40; IC del 95\%, 1.753.30); Asia y Australia: (OR: 1.92; IC del 95\%, 1.602.30) y Europa (Reino Unido únicamente): (OR, 2.76; IC 95\%, 2.48-3.07); las razones de esta asociación aún están en investigación. Debido a limitantes en la información recuperada en nuestro estudio no fue posible realizar análisis inferenciales para explorar esta asociación 18,32,33.

En relación a las características de los afectados estos tenían entre 30 y 39 años de edad, procedían en su mayoría (62.9\%) del área urbana de Tegucigalpa, lo que podría explicarse porque usualmente las personas jóvenes, y residentes en las ciudades son más críticas del sistema sanitario, podrían tener expectativas más altas y se muestren más insatisfechas con los servicios recibidos. Las razones por las cuales los pacientes interponen denuncias son variables, un estudio realizado en Estados Unidos concluyo que la intención a demandar de los pacientes se ve favorecida por la edad, los más jóvenes, denuncian más ( $7.6 \%, p=$ $0.04)$, las personas menos religiosas $(4 \%, p=0.02)$, menos de 15 visitas por año a cualquier médico (7.2\%, $p=0.001)$, estado ambulatorio $(2.4 \%, p=$ 0.02 ), y las mayores expectativas de información se asociaron con una mayor posibilidad de considerar demandar a su médico $(7.6 \%, p=0.002)$. El deseo de los pacientes de revelar un error médico (82.2\%) superó sus expectativas de compensación financiera, particularmente en casos menos graves $(24.1 \%)^{34}$.

Una de las limitaciones importantes en nuestro estudio fue el rastreo de la información, no solo a nivel hospitalario, sino la que fue remitida por la fiscalía, lo que demora en gran manera el proceso de análisis y dictamen.

Los datos analizados no permitieron establecer, qué tipo de arreglo, sanción o pena se derivó a raíz de los dictámenes periciales en los que se concluyó con responsabilidad profesional médica.

En España existe un modelo de Servicio de Responsabilidad Profesional (SRP), modelo centralizado que permite el análisis de los datos sobre errores, eventos adversos y responsabilidad profesional, lo que facilita su prevención, esto ha promovido la seguridad clínica publicando guías de forma periódica, actualizando el conocimiento médico-legal de los facultativos en cuanto a los tipos de responsabilidad que conllevan sus actividades, reconocer cuando están frente a una falla de responsabilidad profesional médica y sus respectivas consecuencias, lo que ha controlado la tendencia al incremento de las reclamaciones gestionando extrajudicialmente los casos, animando a la declaración de eventos adversos y errores, la disculpa y el ofrecimiento de acciones cuando es necesario ${ }^{3,5}$.

\section{Conclusiones:}

Este trabajo representa una aproximación inicial a las denuncias por responsabilidad profesional médica en nuestro país e indica que los médicos más denunciados, son del sexo masculino, de mediana edad (50 años) y con más de 15 años de experiencia, que laboran en centros públicos ejerciendo preferentemente especialidades como ginecobstetricia, ortopedia y cirugía general; los motivos más frecuentes por los que los pacientes o sus familiares denuncian a los médicos son conflictos en la relación médico-paciente-familiar, la inconformidad en el diagnóstico y falta de ética profesional de otros profesionales con comentarios adversos hacia sus colegas o las instituciones.

Desde el punto de vista médico legal únicamente el $5.61 \%$ de las denuncias se dictaminaron con responsabilidad profesional médica. La iatrogenia 
en el $58,33 \%$ de los casos fue un hallazgo importante que debe estudiarse detallada y sistemáticamente.

Se detectaron serias limitantes en el sistema de información y registro de las denuncias, que debe corregirse.

\section{REFERENCIAS BIBLIOGRÁFICAS}

1. Gálvez E, Gálvez M, Díaz M, Morales L. Criterio profesional acerca del error médico. [Internet]. Rev. Cubana Med Gen Integr. 1998 feb [consultado 7 julio 2018]; 14(1): 32-37. Disponible en: http://scielo.sld.cu/scielo.php?script=sci arttext\& pid=S0864-21251998000100005

2. Salazar Torres C. Necesidad de reformar el código orgánico integral penal con la finalidad de establecer la tipificación y penalización de la mala práctica profesional médica. [Tesis Internet]. Loja, Ecuador: Universidad Nacional de Loja; 2015. [consultado 7 julio 2018]. Disponible en: http:// http://dspace.unl.edu.ec:9001/jspui/bitstream/12 3456789/8896/1/Cristhel\%20Dolores\%20Salazar\% 20Torres.pdf

3. Martin Fumadó C. Análisis de la responsabilidad profesional derivada del ejercicio de la Psiquiatría y de la Medicina Legal. [Tesis Internet]. Barcelona: Universidad Autónoma de Barcelona; 2012. [consultado 7 julio 2018]. Disponible en: http://docplayer.es/1990067-Analisis-de-la-

responsabilidad-profesional-medica-derivada-delejercicio-de-la-psiquiatria-y-de-la-medicinalegal.html

4. Organización Mundial de la Salud. Alianza mundial para la seguridad del paciente. La investigación en seguridad del paciente. [Internet]. Ginebra: OMS; $2008 . \quad$ Disponible en: http://www.who.int/patientsafety/es/

5. Carles M. Responsabilidad por una práctica médica inadecuada: una perspectiva económica. Gac Sanit [Internet]. 2003 dic [consultado 22 abril 2018]; 17(6): 494-503. Disponible en: http://scielo.isciii.es/scielo.php?script=sci arttext \&pid=S0213-91112003000600011\&lng=es
6. Ministerio Público, oficina de planificación estratégica y gestión de la calidad. Denuncias de responsabilidad profesional nivel Nacional. Tegucigalpa: Ministerio Público; 2018.

7. Honduras. Congreso Nacional. Corte Suprema de Justicia. Código Penal. Decreto 144-83, actualizado 2014. Tegucigalpa: Congreso Nacional; 2014.

8. Honduras. Congreso Nacional. Corte Suprema de Justicia. Código Civil. Decreto 76-1906. Diario Oficial La Gaceta. 27 de febrero de 2013.

9. Colegio Médico de Honduras. De las sanciones por violaciones de la ética. En: Compendio de leyes y reglamentos del Colegio Médico Honduras [Internet]. Tegucigalpa: $\mathrm{CMH}$; 2005. [consultado 7 julio 2018]; Disponible en: http://www.colegiomedico.hn/servicios/leyes-yreglamentos/

10. Traina F. Medical Malpractice. The Experience in Italy. Clin Orthop Relat Res (2009) 467:434-442. DOI 10.1007/s11999-008-0582-z.

11. Localio AR, Lawthers AG, Brennan TA, Laird NM, Hebert LE, Peterson LM, et al. Relation between malpractice claims and adverse events due to negligence. Results of the Harvard Medical Practice Study III.N EngI J Med. 1991;25;325(4):245-51.

12. Mendoza C, Ramos-Rodríguez C, Gutiérrez E. Relación médico-paciente percibida por usuarios de consultorios externos de un hospital de Lima, Perú. Horiz Med. [Internet]. 2016 [consultado 12 julio 2018];16(1):14-19. Disponible en: http://www.scielo.org.pe/scielo.php?script=sci ar ttext\&pid=S1727-558X2016000100003\&Ing=es

13. Alonso Juste $\mathrm{V}$, Martín Delgado MC, Alegre Martín J, Fernández de Sevilla Ribosa T. Efectos adversos en el enfermo crítico. [Tesis Internet]. Barcelona: Universidad Autónoma de Barcelona; 2016. [consultado 7 julio 2018]; Disponible en: https://ddd.uab.cat/record/168498.

14. Vera Carrasco O. Cómo Prevenir presuntas demandas de mala praxis médica. Rev Méd La Paz [Internet]. 2016 [consultado 12 julio 2018];22(2):60-69. Disponible en: http://www.scielo.org.bo/scielo.php?script=sci_ar ttext\&pid=S1726-89582016000200011\&lng=es 
15. Aguirre Gas HG, Zavala Villavicencio JA, Hernández Torres F, Fajardo Dolci G. Calidad de la atención médica y seguridad del paciente quirúrgico: error médico, mala práctica y responsabilidad profesional. Cir Cir[internet]. 2010[consultado 7 julio 2018]; 78:456-462. Disponible http://www.redalyc.org/html/662/66220238015/ 16. Barrera RN, Jiménez CME, Rodríguez SJG, Victoria OR, Rivera CAE, Manuell LG, et all. Recomendaciones para prevenir la mala práctica Médica en Pediatría. Rev CONAMED [Internet]. 2003[consultado 7 julio 2018]; 8 (1): 15-28. Disponible

en:

http://www.medigraphic.com/pdfs/conamed/con -2003/con031d.

17. Rodríguez Silva H. La relación médico-paciente. Rev Cubana Salud Pública [Internet].2006[consultado 12 julio 2018];32(4). Disponible en:

http://scielo.sld.cu/scielo.php?script=sci arttext\& pid=S0864-34662006000400007\&lng=es

18. Croft E, Clark MT, Efstathiou N, Bradbury-Jones C. A focused mapping review and synthesis of a priori risk factors associated with medical misconduct. BMJ Open Qual. 2019; 8(2): e000538. doi: 10.1136/bmjoq-2018-000538

19. Sardinero -García C, Santiago-Sáez A, PereaPérez $B$, Albarrán-Juan $M E$, Labajo-González $E$, Benito-León J. Responsabilidad por perdida de oportunidad asistencial en la medicina pública española. Rev Esp Med Legal [Internet]. 2017 [Consultado 12 julio 2018 ];43(1):5-12. Disponible en: http://bit.ly/337Lafm

20. Tole HD, Cadavid N. Instrumentador quirúrgico: iatrogenia, eventos frecuentes y responsabilidad en el quirófano. Repert Méd Cir [Internet]. 2018 [Consultado 12 julio 2018];27(2):124-30. Disponible en: https://revistas.fucsalud.edu.co/index.php/repert orio/article/view/185/178

21. Fundación Educación Médica. Documento del grupo de trabajo SESPAS-OMC sobre iatrogenia: Grupo de trabajo SESPAS-OMC sobre iatrogenia.
FEM [Internet]. 2017 [Consultado 12 julio 2018 ];20 (6):255-264. Disponible en: https://www.seepidemiologia.es/documents/dum my/FEM 2006255 C 2517059 SESPAS-OMC.pdf 22. McGuire AR, DeJoseph ME, Gill JR. An approach to iatrogenic deaths Forensic Sci Med Pathol. 2016;12(1):68-80. doi: 10.1007/s12024-016-97455

23. Tena TC. la demanda como efecto de la mala comunicación médico-paciente. Rev CONAMED [Internet]. 2002[Consultado 12 julio 2018 ];7(3):1518. Disponible en:

https://www.medigraphic.com/cgibin/new/resumen.cgi?IDARTICULO=80470

24. Oyebode F. Clinical errors and medical negligence. Med Princ Pract. 2013;22(4): 323-333. doi: 10.1159/000346296.

25. Peña JA. Por qué demandan a los médicos en México y por qué nace el movimiento médico nacional \#yosoy17. Gac Int Cienc Forense [Internet]. 2014[Consultado 12 julio 2018]; 13:5-7. Disponible en: https://www.uv.es/gicf/2TA1 Penya GICF 13.pdf 26. Támara LM, Jaramillo SH, Muñoz LE. Informes periciales por presunta responsabilidad médica en Bogotá. Rev Colomb Anestesiol [Internet]. 2011 [Consultadon21 abril 2018];39(4):489-505. Disponible en: http://bit.ly/2KG9Oxu

27. Perea Pérez B, Santiago Sáez A, Labajo González ME, Albarrán Juan ML, Sánchez Sánchez JA. Análisis de las consecuencias médico-legales de las reclamaciones judiciales de pacientes. Estudio comparativo de los casos de traumatología, ginecología y obstetricia, anestesia y reanimación y odontoestomatología. Trauma Fund MAPFRE [Internet]. 2009 [Consultado 12 julio 2018 ];20(4):264-268. Disponible en: http://www.mapfre.com/fundacion/html/revistas trrauma/v20n4/pdf/02 09.pdf

28. Castellanos Arroyo M, Davide Ferrara S. La responsabilidad profesional médica en Europa. Rev Esp Med legal [Internet]. 2014[Consultado 12 julio 2018];40(4):161-166. Disponible en: 
https://www.sciencedirect.com/science/article/ab s/pii/S0377473214000145

29. Khan IH, Jamil W, Lynn SM, Khan OH, Markland K, Giddins G. Analysis of NHSLA claims in orthopedic surgery Orthopedics [Internet]. 2012 [Consultado 18 de julio 2019]; 35(5):e726-31. Disponible en: http://bit.ly/35s59XV

30. López MA, Manrique J, García C. Demandas profesionales de la salud (observaciones de los casos). [Internet]. Bogotá: III Congreso de derecho médico; 2002[Consultado 12 julio 2018 ]. Disponible en:

http://www.medicolegal.com.co/pdf/esp/2003/9/ $1 / \mathrm{cdm} 2 \mathrm{v} 9 \mathrm{r} 1 . \mathrm{pdf}$

31. Kornmehl H, Singh S, Adler BL, Wolf AE, Bochner DA, Armstrong AW. Characteristics of medical liability claims against dermatologists from 1991 through 2015. JAMA Dermatol. 2018;154(2):16066. doi: 10.1001/jamadermatol.2017.3713
32. Unwin E, Woolf $K$, Wadlow C, Potts HW, Dacre $J$. Sex differences in medico-legal action against doctors: a systematic review and meta-analysis. BMC Med. 2015; 13:172. doi: 10.1186/s12916-0150413-5

33. Unwin E, Woolf K, Wadlow C, Dacre J. Disciplined doctors: does the sex of a doctor matter? A cross-sectional study examining the association between a doctor's sex and receiving sanctions against their medical registration. BMJ Open. 2014;4(8):e005405. doi: 10.1136/bmjopen2014-005405

34. Tsimtsiou Z, Kirana P, Hatzimouratidis K, Hatzichristou D. What is the profile of patients thinking of litigation? results from the hospitalized and outpatients' profile and expectations study. Hippokratia [Internet]. 2014 [Consultado 12 julio 2018 ];18 (2):139-143. Disponible en: https://www.ncbi.nlm.nih.gov/pmc/articles/PMC4 201400/1. 